\title{
Cryo-FIB for Thinning Cryo-TEM Samples and Evading Ice During Cryo-transfer
}

\author{
W. J. MoberlyChan*, M. Marko**, and C.-E. Hsieh** \\ *Harvard University, DEAS \& CIMS, Cambridge, MA 02138. **Resource for Visualization of \\ Biological Complexity, Wadsworth Center, Empire State Plaza, Albany, NY 12201.
}

When a Gatan Alto 2500 cryo stage is attached to an FEI-DualBeam DB235 FIB/SEM, the process of FIBsectioning sample preparation for TEM can be extended to Cryo-FIB-TEM prep [1]. The possibility now exists to use FIB for submicron site-specific sectioning inside biological materials such as cells. The practice remains less productive than cryo-ultramicrotomy; however, using the SEM inside the DB235 enables focusing the prep on nanometer-scale features in the sample. The devil in the details is not the fire (heat of either of the dual beams) but rather the ice (frost) that can occur at each step of the cryo-transfer-process. Until an allencompassing cryo-fast-freeze-prethin-FIB-SEM-TEM single tool is available, cryo-transfer is a necessary evil. To test the cryo-FIB-prep viability we have pushed the limits of cryo-transfer. A cryo sample is plunge-frozen at Albany, cryo-transferred to Harvard and into (and out of) the cryo FIB using a modified specimen holder, then cryo transferred back to Albany for cryo-TEM [2]. After several cryo-transfers from one holder to another, frost is difficult to avoid. However, with a goal to study submicron features, the cryo-DB235 enables one to ignore many ice boulders. FIB ion milling (with $30 \mathrm{kV}$ Ga ions) provides etching rates of ice that are $>100$ times that of Si [3]. Because the high-energy ions can penetrate deep into ice-based materials, care must be taken to avoid ion-induced artifacts [1]. However, the grazing incident ion beam used in this work for thinning limits damage, and FIB can be performed without generating heat and without crystallizing vitreous ice [2].

Two sample types are considered. The ultimate may be to FIB-section into a large block of material and then "lift-out" a cryo-section for the TEM. However, "lift-out" methods remain difficult. Pt-welding at low temperature to an Ominprobe is impractical to control, although alternate materials may help; and other cryoFIB-biopsies "lift-out" methods can be successfully performed with the Omniprobe. Since the difficulty of "liftout" has a pay-off for room temperature materials in that it can be treated as non-destructive for the rest of a 12inch wafer, "lift-out" may be less valuable to cryo-FIB-bio-prep, as the cell will not be asked to perform live functions after the cryo-FIB-TEM-prep. The easier problem, and more valuable site-specific cryo-FIB-prep, may be to take an already relatively thin cryo specimen and provide extra local thinning to enhance TEM analysis. Some plunge-frozen TEM samples may be too thick (or covered by frost) for viewing of nanometerscale features. Thus cryo-FIB-TEM prep to further thin an area is of use.

Figures 1a and $1 \mathrm{~b}$ are SEM images acquired before and after FIB thinning. The cryo-TEM samples have been plunge-frozen on a Quantifoil thin carbon membrane with holes. The TEM grid was then cut in half so that the edge could be FIBed on either front and/or back sides. Areas of large ice crystals from cryo-transfer procedures were avoided. A region at the left was used for aligning the FIB beam. After two areas (arrows) are thinned by grazing-incidence FIB etching, they appear "brighter" in SEM imaging due to thinned areas allowing more penetration of the electron beam. Fig. 2 is an SEM image of trenches produced with a 30kV-10pA ion beam in $~ 10$ seconds. Fig. 3 is a FIB image (secondary electron detector) depicting a region thinned at high magnification. At low magnification, the sample can be FIB-imaged with negligible damage, and contrast in the FIB image (Fig. 4) can help determine when thick ice on the sample is getting thinner.

\section{References}

[1] H. Mulders G.I.T. Imaging and Microscopy 2 (2003) 8.

[2] M. Marko, et al. Microsc. Microanal. 11 (suppl.2) (2005) this proceedings.

[3] W. MoberlyChan, et al. Microsc. Microanal. 11 (suppl.2) (2005) this proceedings.

[4] Supported by NSF NINN at Harvard and NIH NCRR RR01219 at Wadsworth Center. 

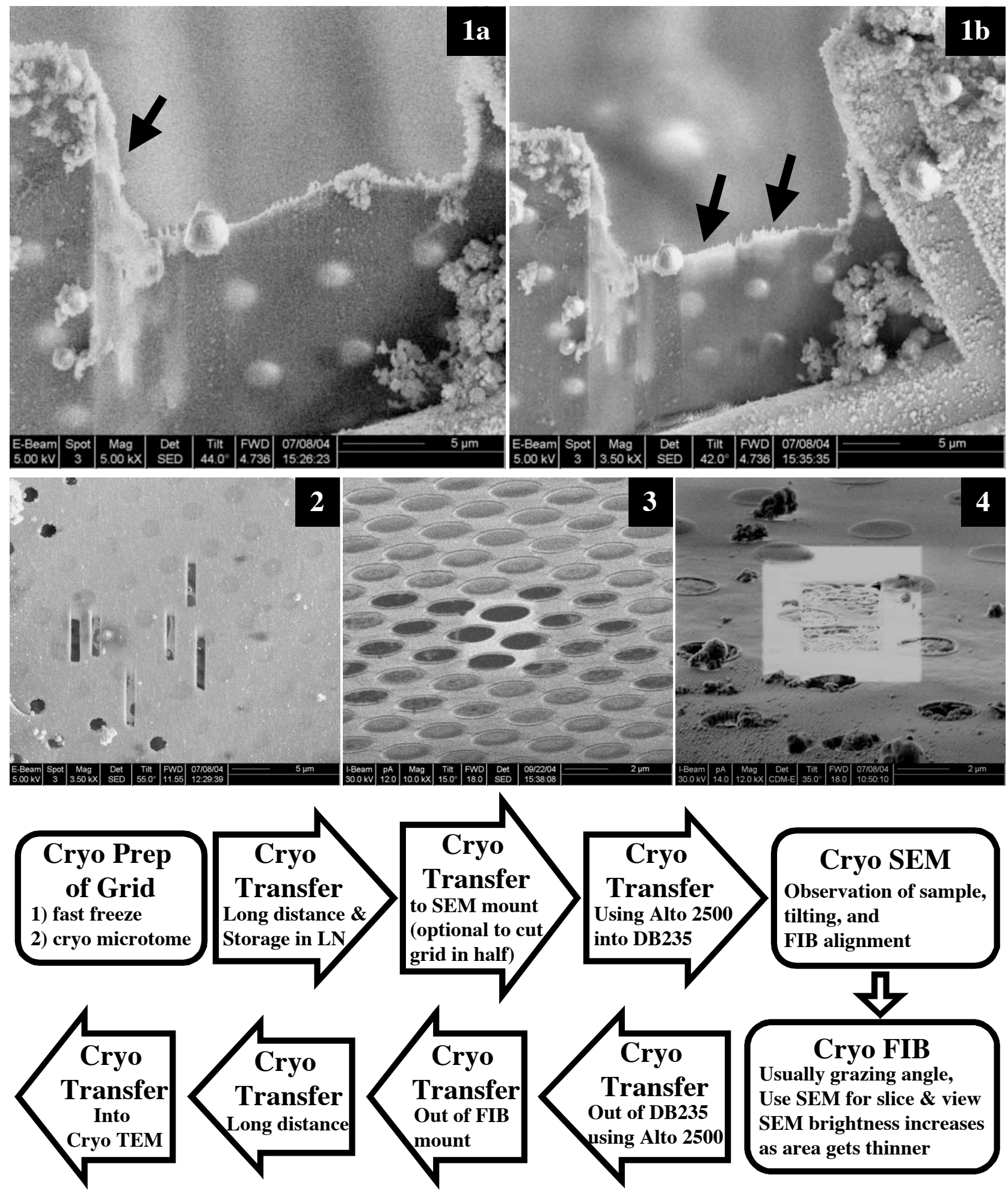

FIG. 1. SEM before and after FIB thinning cryo-TEM sample on $\mathrm{Cu}$ grid cut in half.

FIG. 2. SEM image of grazing FIB etch-trenches of cryo-TEM sample on Quantifoil grid.

FIG. 3. FIB image of area of Quantifoil TEM grid thinned by FIB etching.

FIG. 4. FIB image of thinned Quantifoil sample that had a thick ice $(>200 \mathrm{~nm})$. 